\title{
Hepatotoxicidad secundaria a "productos naturales": análisis de los casos notificados al Registro Español de Hepatotoxicidad
}

\author{
M. García-Cortés ${ }^{1 a}$, Y. Borraz ${ }^{2 a}$, M. I. Lucena ${ }^{2 a}$, G. Peláez ${ }^{3}$, J. Salmerón ${ }^{4 a}$, M. Diago ${ }^{5}$, M. C. Martínez-

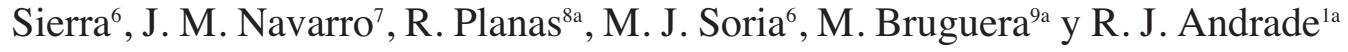 \\ ${ }^{1}$ Unidad de Hepatología. Servicio de Aparato Digestivo. ${ }^{2}$ Servicio de Farmacología Clínica. Hospital Universitario \\ Virgen de La Victoria. Facultad de Medicina. Málaga. ${ }^{3}$ Servicio de Aparato Digestivo. Hospital Torrecárdenas. Almería. \\ ${ }^{4}$ Servicio de Aparato Digestivo. Hospital Universitario San Cecilio. Granada. ${ }^{5}$ Servicio de Aparato Digestivo. Hospital \\ General. Valencia. ${ }^{6}$ Servicio de Aparato Digestivo. Hospital Puerta del Mar. Cádiz. ${ }^{7}$ Servicio de Aparato Digestivo. \\ Hospital Costa del Sol. Marbella, Málaga. ${ }^{8}$ Servicio de Aparato Digestivo. Hospital Germans Trías y Pujol. Badalona, \\ Barcelona.${ }^{9}$ Unidad de Hepatología. Hospital Clínic. Barcelona. ${ }^{a}$ Centro de Investigación Biomédica en Red de \\ Enfermedades Hepáticas y Digestivas (CIBERehd)
}

\section{RESUMEN}

Introducción: la toxicidad hepática asociada al uso creciente de productos de "remedios naturales" es un fenómeno emergente.

Objetivos: valoración de las características epidemiológicas, clínicas y demográficas de los casos de hepatotoxicidad secundarios a productos herbales (PH) y suplementos dietéticos (SD).

Pacientes y métodos: análisis de los casos de hepatotoxicidad debida a PH y SD incluidos en el Registro Español de Hepatotoxicidad.

Resultados: trece casos de un total de 521 casos (2\%) de reacciones adversas hepáticas incluidas en el registro entre 1994 y 2006, eran secundarios a PH/SD, representando el décimo grupo terapéutico responsable por orden de frecuencia, por delante de analgésicos, ansiolíticos y antipsicóticos. Nueve pacientes (69\%) eran mujeres y la edad media fue de 45 años. Nueve pacientes (69\%) presentaron ictericia. El tipo de daño más frecuente fue el hepatocelular $(12 ; 92 \%)$ y un $31 \%$ de los casos presentaron datos de hipersensibilidad. La sustancia más comúnmente involucrada en los casos de daño hepático fue la Camellia sinensis (23\%) seguida de Rhamnus purshianus e isoflavonas (Fitosoja ${ }^{\circledR}$, Biosoja ${ }^{\circledR}$ ) con dos casos cada uno (15\%). Tres casos (23\%) presentaron re-exposición positiva.

Conclusiones: la hepatotoxicidad originada por PH/SD no es excepcional, y su perfil es la hepatitis aguda hepatocelular ictérica predominantemente en mujeres. La frecuente ocurrencia de reexposición positiva en estos pacientes indica un bajo índice de sospecha y un retraso o ausencia de diagnóstico de este tipo de reacción adversa.

Palabras clave: Hepatotoxicidad. Reacciones adversas hepáticas. Productos herbales. Medicina natural.

Recibido: 11-08-08.

Aceptado: 02-09-08.

Correspondencia: Raúl J. Andrade Bellido. Departamento de Medicina. Facultad de Medicina. Boulevard Louis Pasteur, 32. 29071 Málaga. e-mail: andrade@uma.es

\section{ABSTRACT}

Background: toxic liver damage associated with the use of natural remedies is a growing health problem.

Objectives: to analyze the demographics, and clinical and epidemiological characteristics of patients developing liver injury related to these remedies.

Patients and methods: all DILI cases associated with the use of herbal remedies (HR) or dietary supplements (DS) submitted to the Spanish Registry were analyzed. Type of liver damage, severity, and outcome were specifically evaluated.

Results: thirteen cases out of 521 DILI cases (2\%) submitted to the Spanish Liver Toxicity Registry between 1994 and 2006 were related to HR/DS, which ranked as the $10^{\text {th }}$ therapeutic group with a greater number of cases and above pain killers, anxiolytics, and antipsychotic drugs. Nine patients (69\%) were female (mean age 45 years). Nine cases (69\%) had jaundice at presentation. The predominating type of liver damage was hepatocellular $(12 ; 92 \%)$, and $31 \%$ of cases exhibited the common features of hypersensitivity. Camellia sinensis (3, $23 \%$ ) was the main causative herb, followed by Rhamnus purshianus and isoflavones (Fitosoja ${ }^{\circledR}$, Biosoja ${ }^{\circledR}$ ) (2 cases each, $15 \%)$. Three cases (23\%) were rechallenged with the offending product.

Conclusions: the incidence of hepatic damage related to $\mathrm{HR} / \mathrm{DS}$ is not so rare, the most common profile of affected patients being a woman with acute hepatocellular hepatitis. Low suspicion regarding the putative role of herbs in hepatotoxicity makes diagnosis more difficult, and probably increases the incidence of inadvertent rechallenge in these patients.

Key words: Hepatotoxicity. Reacciones adversas hepáticas. Productos herbales. Natural remedies.

Agradecimientos y financiación: Agradecemos la participación de D. Ramón Hidalgo en el análisis estadístico de los datos. Este estudio ha sido parcialmente financiado con una ayuda de la Agencia Española del Medicamento y con una beca FIS 07/0980. CIBERehd está financiado por el Instituto de Salud Carlos III. 
García-Cortés M, Borraz Y, Lucena MI, Peláez G, Salmerón J, Diago M, Martínez-Sierra MC, Navarro JM, Planas R, Soria MJ, Bruguera M, Andrade RJ. Hepatotoxicidad secundaria a "productos naturales": análisis de los casos notificados al Registro Español de Hepatotoxicidad. Rev Esp Enferm Dig 2008; 100: 688-695.

\section{INTRODUCCIÓN}

En las últimas décadas estamos viviendo un incremento de la popularidad de la medicina natural en los países desarrollados. En el marco de la medicina alternativa, el consumo de productos botánicos es el que mayor auge ha alcanzado y el que mayor incremento ha experimentado en las últimas décadas, estimándose una prevalencia actual entre un 18,6 y el $37 \%$ con amplias variaciones entre distintos países y tipos de estudios (1-3). Un estudio realizado en nuestro país señalaba que uno de cada cinco pacientes en tratamiento con medicamentos consumía hierbas medicinales de forma concomitante (4).

Este aumento en el consumo de productos herbales es debido principalmente a la percepción de que al ser "naturales" sólo pueden ser beneficiosos y carecen de riesgos para la salud. Sin embargo, aunque hoy día sabemos que las sustancias de origen vegetal no carecen de efecto biológico, las propiedades de las mismas están escasamente estudiadas y contrastadas (5). Es más, existe una falta de regulación de los compuestos botánicos en muchos países como Estados Unidos, en los que estos productos se consideran como suplementos dietéticos, escapando así de las exigencias aplicadas en materia de eficacia y seguridad a los fármacos convencionales (6). Además, se carece de programas específicos de vigilancia post-comercialización, por lo que la incidencia y características de los efectos adversos que producen son desconocidos. Sin embargo, comienza a emerger la evidencia del riesgo de toxicidad asociada a una amplia variedad de estos productos en los últimos años, siendo la causa más frecuente de hepatotoxicidad en los países asiáticos (7).

Recientemente el Gobierno español junto con la Agencia Española de Farmacovigilancia, han publicado una lista de plantas cuya venta al público fuera de los establecimientos sanitarios y al margen de la legislación sobre medicamentos queda prohibida o restringida debido a la toxicidad demostrada (OM 190/2004, BOE 32 febrero 2004). Por desgracia, el escaso control sobre los preparados que se venden en herbolarios y tiendas de dietética no permite descartar que se sigan vendiendo algunas de las especies prohibidas. En este listado, los productos que han sido relacionados con episodios de hepatotoxicidad son los siguientes: extracto etanólico de Camelia Sinensis $\left(\right.$ Exolise $\left.^{\circledast}\right)$, Actracilys gummifera, celandina mayor, crotalaria, efedra, heliotropium, kava-kava, cáscara sagrada, senecio, Teucrium chamaedrys (camedrio), la consuelda y el Viscus album.
En el presente trabajo se han analizado las características clínicas y demográficas de los casos de hepatotoxicidad secundaria a PH/SD incluidos en el Registro Español.

\section{PACIENTES Y MÉTODOS}

Los casos analizados pertenecen al Registro Español de Hepatotoxicidad, una base de datos no restringida y que captura casos de toxicidad hepática debida a fármacos o tóxicos, fundado en 1994 y coordinado por dos de los autores (R.J.A y M.I.L). Para la recogida de datos se utilizó un protocolo estructurado que contiene la siguiente información: la relación temporal entre el comienzo del consumo del fármaco o la exposición al tóxico y el inicio de la enfermedad hepática y entre la suspensión del agente sospechoso y la mejora o recuperación de la disfunción hepática; descartar otras enfermedades hepáticas; presencia de factores de riesgo conocidos de hepatotoxicidad como el consumo de alcohol (cantidad en conversión del volumen de bebida en gramos) o embarazo; y el resultado del daño hepático. Se revisaron exhaustivamente los fármacos de consumo actual y los previos, $\mathrm{PH} / \mathrm{SD}$ y posibles tóxicos. Se excluyeron otras causas de enfermedad hepática: etílica, hepatitis viral reciente por virus A (IgM antiVHA), B (IgM antiVHB) o C (antiVHC y PCR), enfermedades autoinmunes (ANA, anticuerpos antimitocondriales, anti-LKM1 y anti-músculo liso) y obstrucción biliar (ecografía abdominal complementada con resonancia magnética o colangiografía endoscópica en los casos necesarios). Si el contexto clínico lo sugería se descartó citomegalovirus, Epstein Barr, infección por herpes virus o hepatitis E y serología de bacterias como Salmonella, Campylobacter y listeria. Si el paciente era menor de 40 años se determinó la ceruloplasmina y la excreción urinaria de cobre para descartar la enfermedad de Wilson. Se evaluó asimismo la presencia de otras enfermedades metabólicas hepáticas como la hemocromatosis, el déficit de alfa 1 antitripsina y en pacientes con historia de hipotensión reciente se descartó la presencia de hepatitis isquémica. La indicación de biopsia hepática queda a criterio del médico notificador del caso y se restringe contextos dudosos, como pacientes con marcadores de autoinmunidad, alcohólicos, enfermedad hepática previa o enfermedades sistémicas que pueden afectar al hígado se indicó una biopsia hepática para obtener más información etiológica. 
Todos casos remitidos al registro fueron revisados por el médico responsable y posteriormente evaluados por tres expertos independientes del centro coordinador quienes valoraron la causalidad, primero por juicio clínico y posteriormente mediante la aplicación de la escala Council for Internacional Organizations of Medical Sciences (CIOMS/RUCAM) $(8,9)$. Dicha escala se basa en un sistema de puntuación estandarizado según diferentes criterios (cronológicos, evolución, factores de riesgo, fármaco concomitante, exclusión de causas alternativas no farmacológicas, información previa de hepatotoxicidad del fármaco, reexposición), finalmente el resultado se traslada a categorías de sospecha divididas en: definida o altamente probable ( $>8$ puntos), probable (6-8 puntos), posible (3-5 puntos), improbable ( $<2$ puntos) y excluida ( $\leq 0$ puntos). Únicamente los casos valorados por la escala CIOMS como posible, probable o definido se incluyeron en la base de datos.

La definición y clasificación del tipo de lesión fueron establecidas mediante la aplicación de los criterios derivados de la reunión del Grupo Internacional de Consenso (10).

Se definió lesión hepática al incremento de dos veces sobre el límite superior a la normalidad (LSN) en alanino aminotransferasa (ALT) o bilirrubina conjugada; o a la combinación del incremento de aspartato aminotransferasa (AST), fosfatasa alcalina (FA) y bilirrubina total siendo uno de ellos 2 veces $>$ LSN. Se clasificó el tipo de lesión en base a los hallazgos anatomopatológicos o alternativamente en base a los hallazgos bioquímicos si no se disponía de biopsia. El daño hepático se determinó como hepatocelular cuando el ratio ALT/FA era $\geq 5$, como colestásica $\leq 2$ y mixta $>2$ pero $<5$. Se utilizaron para dicha clasificación los primeros valores analíticos realizados al paciente. Finalmente se intentó establecer el mecanismo patogénico del daño hepático clasificándolo en intrínseco o idiosincrásico, y este último en inmunoalérgico si presentaba datos de hipersensibilidad.

Los datos obtenidos fueron analizados con el paquete estadístico SPSS (Statiscal package for the Social Sciences, versión 14.0 para Windows).

\section{RESULTADOS}

De un total de 521 casos de hepatotoxicidad incluidos en el Registro Español de Hepatotoxicidad entre los años 1994 y 2006, se detectaron 13 casos de hepatotoxicidad secundaria a $\mathrm{PH} / \mathrm{SD}$, lo cual corresponde a un $2 \%$ de los casos representando el décimo grupo terapéutico responsable por orden de frecuencia, por delante de analgésicos, ansiolíticos y antipsicóticos. En la tabla I se describen los datos demográficos y clínicos de los casos incluidos en la presente serie. Dos de los casos incluidos en esta revisión habían sido previamente publicados $(11,12)$. Las sustancias responsables de los casos de hepatotoxicidad en el registro fueron Camellia sinensis (té verde) con tres ca- sos, Rhamnus purshianus (cáscara sagrada) con dos casos y Aesculus hippocastanum (castaño de indias), Couterea latifloral (Copalchi), Chitosan, Cassia angustifolia (senna), valeriana, Piper methysticum rizoma (Kava-kava), fitosoja y biosoja con un caso cada uno.

La indicación terapéutica más frecuente de estas sustancias fue la obesidad en seis casos. En el resto de los pacientes, las sustancias vegetales fueron ingeridas para el alivio de los síntomas de la menopausia, ansiedad, estreñimiento, dispepsia, insuficiencia venosa periférica, diabetes mellitus y como reconstituyente.

La edad media fue de 45 años (23-78) y nueve pacientes correspondían al sexo femenino $(69 \%)$. La duración media del tratamiento fue de 94 días (1-540 días) y el periodo de latencia entre el inicio del tratamiento y el comienzo de la enfermedad fue de 79 días (1-365 días). El motivo de consulta más frecuente fue la ictericia, síntoma que apareció en nueve pacientes (69\%). En el resto de los pacientes se identificó el problema al detectar hipertransaminasemia. Cuatro pacientes $(31 \%)$ presentaron datos de hipersensibilidad y el tipo de lesión hepática más frecuente en la presente serie fue el daño hepatocelular en 12 casos $(92 \%)$. Se realizó biopsia hepática en tres pacientes de la serie obteniendo resultados compatibles con hepatitis tóxica en todos ellos (Tabla I).

En tres de los casos descritos hubo una reexposición positiva, es decir, se evidenció una recidiva de la enfermedad hepática tras la readministración de la sustancia responsable de la lesión hepática. Dicha reexposición fue accidental en todos los casos, debido fundamentalmente a la ausencia de sospecha o diagnóstico del episodio índice.

En cuanto a la gravedad y la evolución del cuadro, seis pacientes precisaron hospitalización (46\%) y no se produjeron fallecimientos por esta causa. Una paciente requirió ser derivada a un hospital con programa de trasplante hepático que finalmente no requirió por mejoría espontánea de la insuficiencia hepática. La resolución completa del cuadro se apreció en once pacientes, un paciente presentó persistencia en la elevación de la GGT y finalmente, un paciente presentó datos de cronicidad con signos de fibrosis en la biopsia hepática.

La aplicación de la escala internacional de evaluación de causalidad en hepatotoxicidad derivó en una categoría de probabilidad definida o altamente probable en 6 pacientes $(46 \%)$ y en probable en 7 pacientes $(54 \%)$.

\section{DISCUSIÓN}

El hígado es el principal órgano implicado en el metabolismo de cualquier sustancia ajena al organismo incluidos los productos botánicos, lo cual hace que sea especialmente susceptible a la toxicidad por dichas sustancias. Contrariamente a la creencia popular, lejos de ser seguros, los remedios herbales comportan un riesgo de toxicidad hepática en algunos casos con consecuencias fatales (13). Estos compuestos "naturales" han sido 
Tabla I. Datos demográficos y clínicos de los casos con hepatotoxicidad secundaria a productos de herboristería

\begin{tabular}{|c|c|c|c|c|c|c|c|c|c|c|c|c|}
\hline \multirow[t]{2}{*}{ SexolEdad } & \multirow[t]{2}{*}{ Sustancia } & \multirow[t]{2}{*}{ Presentación } & \multirow[t]{2}{*}{ Duración } & \multirow[t]{2}{*}{$\begin{array}{l}\text { po de } \\
\text { latencia }\end{array}$} & \multicolumn{4}{|c|}{$\begin{array}{l}\text { Datos bioquímicos (xLSN) } \\
\text { Episodio agudo/Final seguimiento }\end{array}$} & \multirow[t]{2}{*}{$\begin{array}{l}\text { Patrón de daño } \\
\text { hepático }\end{array}$} & \multirow[t]{2}{*}{ Resolución } & \multirow[t]{2}{*}{ Comentarios } & \multirow[t]{2}{*}{$\begin{array}{l}\text { CIOMs } \\
\text { RUCAM }\end{array}$} \\
\hline & & & & & BrTot & ALT & $F A$ & GGT & & & & \\
\hline$F / 23$ & Camellia sinensis & Ictericia & 21 & 19 & $11,5 / 0,4$ & $56,9 / 0,35$ & $0,88 / 0,20$ & $0,9 / 0,36$ & Hepatocelular & $3 m$ & $\begin{array}{l}\text { Hospitalización } \\
\text { Reexposición }\end{array}$ & Definido \\
\hline M/78 & Casia angustifolia & Ictericia & 161 & 150 & $11,7 / 0,9$ & $35,5 / 0,55$ & $1,4 / 1,1$ & $2,3 / 0,44$ & Hepatocelular & $1 \mathrm{~m}$ & $\begin{array}{l}\text { Hospitalización } \\
\text { Alcohol } 10 \mathrm{~g} / \mathrm{d}\end{array}$ & Probable \\
\hline$F / 34$ & Kava & $\begin{array}{l}\text { Hipertransa- } \\
\text { minasemia }\end{array}$ & 152 & 150 & $0,5 / 0,15$ & $18 / 0,8$ & $1,2 / 0,9$ & $4,3 / 0,7$ & Hepatocelular & $14 \mathrm{~m}$ & $\begin{array}{l}\text { VHCt } \\
\text { Reexposición }\end{array}$ & Probable \\
\hline M/55 & Valeriana & Ictericia & 1 & 1 & $16,9 / 0,36$ & $0,6 / 0,71$ & $0,9 / 0,71$ & $2,1 / 0,8$ & $\begin{array}{l}\text { AP: colestasis } \\
\text { centrolobulillar y } \\
\text { discreta fibrosis }\end{array}$ & $6 \mathrm{~m}$ & $\begin{array}{l}\text { Hospitalización HS } \\
\text { AML } 1 / 320\end{array}$ & Definido \\
\hline$F / 27$ & Camellia sinensis & Ictericia & 17 & 5 & $11,4 / 0,85$ & $83,5 / 0,88$ & $1,67 / 0,65$ & $4,5 / 0,74$ & Hepatocelular & $1,5 \mathrm{~m}$ & & Probable \\
\hline$F / 26$ & Camellia sinensis* & Ictericia & 121 & 121 & $16,6 / 8,1$ & $46,4 / 32,1$ & $1,1 / 1$ & $2,8 / 2,4$ & $\begin{array}{l}\text { AP: necrosis } \\
\text { hepatocitos, fibrosis } \\
\text { centrolobulillar y } \\
\text { eosinófilos. } \\
\text { Neoformación de } \\
\text { conductos biliares }\end{array}$ & Cronificación & $\begin{array}{l}\text { Hospitalización } \\
\text { Datos HS } \\
\text { ANA 1/40 } \\
\text { Reexposiciónt }\end{array}$ & Definido \\
\hline$M / 44$ & Rhamnus purshianus & Ictericia & 63 & 63 & $7,1 / 1,5$ & $56,1 / 0,5$ & $0,8 / 0,4$ & $4,6 / 0,3$ & Hepatocelular & $3 m$ & Datos HS & Probable \\
\hline$F / 32$ & Fitosoja & Ictericia & 31 & 23 & $0,53 / 0,50$ & $3,2 / 0,7$ & $0,52 / 0,58$ & $0,28 / 0,2$ & Hepatocelular & $7 \mathrm{~m}$ & & Probable \\
\hline$F / 36$ & Rhamnus purshianus & Ictericia & 17 & 25 & $14,2 / 1,1$ & $54,4 / 0,7$ & $0,57 / 0,26$ & $4,3 / 1,7$ & Hepatocelular & Persiste GGT & $\begin{array}{l}\text { Datos HS } \\
\text { ANA 1/40 } \\
\text { AMA 1/40 } \\
\text { VIH y VHC }\end{array}$ & Probable \\
\hline$F / 57$ & Biosoja & $\begin{array}{l}\text { Hipertransa- } \\
\text { minasemia }\end{array}$ & 59 & 71 & & $9,5 / 0,82$ & $0,9 / 0,5$ & $2,1 / 0,8$ & Hepatocelular & $2 m$ & $\begin{array}{l}\mathrm{Tt}^{0} \text { sinvastatina, } \\
\text { dos sustancias } \\
\text { imputadas }\end{array}$ & Probable \\
\hline$F / 69$ & $\begin{array}{l}\text { Aesculus } \\
\text { Hippocastanum }\end{array}$ & Ictericia & 29 & 29 & $3,8 /-$ & $11,6 / 4,2$ & $2 / 1,9$ & $9,8 / 8,8$ & Hepatocelular & & Hospitalización & Definido \\
\hline$F / 42$ & Chitosan & $\begin{array}{l}\text { Hipertransa- } \\
\text { minasemia }\end{array}$ & 3 & 1 & $0,8 / 0,6$ & $25,1 / 16$ & - & - & Hepatocelular & & Hospitalización & Definido \\
\hline M/64 & $\begin{array}{l}\text { Couterea latifloral } \\
\text { (Copalchil) }^{*}\end{array}$ & $\begin{array}{l}\text { Hipertransa- } \\
\text { minasemia }\end{array}$ & 540 & 365 & $0,7 / 0,8$ & $3,2 / 1,1$ & $0,6 / 0,6$ & $0,8 / 0,5$ & Hepatocelular & $2 m$ & Alcohol $8,5 \mathrm{~g} / \mathrm{d}$ & Definido \\
\hline
\end{tabular}

ALT: alanino aminotransferasa; AMA: anticuerpos antimitocondriales; AML: anticuerpos anti-músculo liso; ANA: anticuerpos antinucleares; AP: anatomía patológica; AST: aspartato aminotransferasa; Br: bilirrubina total; F: femenino; FA: fosfatasa alcalina; HS: hipersensibilidad; M: masculino; LSN: límite superior a la normalidad; VHC: virus de la hepatitis C; VIH: virus de la inmunodeficiencia humana. ${ }^{*}$ Casos publicados $(10,11)$.

relacionados con distintos cuadros de enfermedad hepática, desde hepatitis aguda o crónica, cuadros de colestasis y lesiones vasculares hasta episodios de insuficiencia hepática aguda $(14,15)$. Es más, un estudio en EE. UU. puso de manifiesto que los productos de herboristería eran responsables de un amplio porcentaje de casos derivados para trasplante hepático debido al desarrollo de fallo hepático fulminante (16).

Los factores que pueden contribuir a la hepatotoxicidad de los productos de herboristería son el efecto tóxico de la/s sustancia/s del mismo, la falta de identificación del producto y de sus componentes, la selección de una parte errónea de la planta, el almacenamiento inadecua- do, la contaminación de las plantas por productos químicos, metales pesados o microorganismos, la adulteración durante la producción y la falta o el mal etiquetado de los productos $(17,18)$. Por lo tanto, establecer el diagnóstico de hepatitis tóxica por uso de productos de herboristería, muchas veces de consumo no reconocido, es complicado (19). Sus efectos son, a menudo, crónicos, inespecíficos y clínicamente inaparentes durante tiempo. A pesar de la infra-notificación existente con este tipo de terapias, más de 5.000 reacciones adversas secundarias a hierbas habían sido notificadas a la OMS antes de 1996 y un total de 2.621 eventos habían sido remitidos a la FDA entre los años 1993 y 1998 (20). 
En realidad, la descripción de toxicidad con RH/SD es un fenómeno en constante crecimiento en los últimos años. Entre 2004-2007 creció la representación relativa de los casos atribuidos a este tipo de productos en el Registro Español de Hepatotoxicidad, respecto al análisis correspondiente a los años 1994-2004 (21) (datos no publicados).

Un ejemplo reciente de toxicidad por productos de la medicina natural es lo ocurrido con Herbalife ${ }^{\circledast}$, un suplemento nutricional, con el que se han descrito casos de hepatotoxicidad en diferentes países. En Israel se han publicado 12 casos y 10 en Suiza $(22,23)$. De estos 22 pacientes, dos presentaron fallo hepático fulminante y 5 tuvieron readministración positiva (24). Por último, en España se han notificado un total de 11 casos de daño hepático secundario a este producto al Sistema Nacional de Farmacovigilancia, algunos de ellos han sido publicados en la literatura $(25,26)$. Dado que la composición de los productos Herbalife ${ }^{\circledast}$ es muy diversa y que cada paciente consumía varios compuestos de la marca, es difícil determinar la causa exacta y el mecanismo de la toxicidad. Los posibles mecanismos son la presencia de sustancias conocidas como hepatotóxicas en algunos de dichos productos (Camellia sinensis y concentrados de aloe vera), la interacción entre distintos ingredientes y la contaminación o adulteración de los productos.

Los casos descritos en la presente serie presentaban una secuencia temporal compatible, tanto en la aparición de la reacción adversa como en la mejoría de la misma tras la suspensión de la sustancia imputada. Además se excluyeron otras causas de alteración hepática y hubo una reexposición positiva en tres casos reforzando el diagnóstico de hepatotoxicidad.

$\mathrm{Al}$ igual que lo descrito en publicaciones previas, en el presente estudio se aprecia un predominio del sexo femenino. Dicho predominio clásicamente se ha atribuido a un mayor riesgo o susceptibilidad al desarrollo de episodios de hepatotoxicidad por parte de las mujeres $(13,27)$. Dado que dicha afirmación se ha puesto en entredicho en estudios epidemiológicos recientes, no queda claro si la mayor frecuencia de hepatotoxicidad por productos naturales es debida a un mayor consumo o a una mayor susceptibilidad del sexo femenino. En todo caso el perfil prototípico de los pacientes de esta serie fue el de mujeres con hepatitis hepatocelular ictérica. Este perfil en el contexto de una reacción hepatotóxica es de riesgo para una evolución fulminante como se ha demostrado en un estudio reciente (21). Conviene resaltar que en la mayoría de los casos la indicación de este tipo de productos era el tratamiento de la obesidad debido a sus propiedades adelgazantes o el alivio de síntomas como el estreñimiento o la ansiedad. La reducción del peso corporal se ha convertido en los países avanzados como un objetivo de gran parte de la población, ya sea por razones estéticas o de salud, para lo cual un número no despreciable de personas recurren a la medicina natural y productos milagro en lugar de seguir las recomendaciones dietéticas y de ejercicio físico (28).
El elevado índice de reexposición en esta serie (23\%) en comparación con el total de los casos incluidos en el registro $(6 \%)$ podría deberse no sólo a la percepción de inocuidad de estas sustancias por parte de sus consumidores sino también a la escasa sospecha clínica existente por parte de los profesionales sanitarios sobre la posibilidad de la responsabilidad de los productos herbales en el desarrollo de la enfermedad hepática, lo cual deriva en la falta de diagnóstico y en la imposibilidad de prevenir o evitar nuevos episodios secundarios a la readministración de dichas sustancias $(29,30)$.

En cuanto a las sustancias implicadas en reacciones adversas hepáticas de la presente serie, algunas han sido ampliamente relacionadas con este tipo de enfermedad. Hasta 2007 han sido publicados más de 30 casos de hepatotoxicidad secundaria a té verde (Camellia Sinensis) ingeridos con fines adelgazantes, uno de ellos perteneciente al presente estudio $(11,31-43)$. Es más, una especialidad farmacéutica que contenía el extracto etanólico de esta sustancia, Exolise ${ }^{\circledast}$, fue retirada del mercado por la Agencia Española del Medicamento y productos farmacéuticos en abril de 2003 debido a la notificación de 4 casos a la Agencia Española de Farmacovigilancia y 9 casos notificados en Francia (44). Comúnmente se ha atribuido hepatotoxicidad a su extracto etanólico y en menor medida a la «camilina» o polvo de hojas de té trituradas (Ar$\left.\operatorname{kocapsulas}^{\circledR}\right)$. Sin embargo, desde su retirada, se han seguido comunicando casos de daño hepático secundario a otras formas de Camellia sinensis como las infusiones $(33,34)$.

Las reacciones adversas hepáticas secundarias a la ingesta de cáscara sagrada (Rhamnus purshiana), también han sido descritas en la literatura y han sido declarados como ilegales por la agencia española del medicamento algunos productos que contienen dicha sustancia. Uno de los ingredientes de este remedio son los glucósidos antracénicos, previamente descritos como causantes de lesión hepática crónica (45). El caso de hepatotoxicidad secundario a Copalchi está publicado previamente junto con otros 4 casos, habiéndose encontrado además otras seis notificaciones en la literatura (12).

El Piper methysticum o kava-kava, crece fundamentalmente en zonas de la Polinesia y se utiliza para el tratamiento de trastornos ansioso-depresivos debido a la actividad sedativa de las sustancias kavapironas, agonistas de los receptores GABA (46). En varios países europeos, entre los que se encuentra España se prohibió la comercialización de los productos que contuvieran esta planta debido a la comunicación de más de 20 casos de toxicidad hepática; posteriormente dicha retirada se extendió al resto de países de la Unión Europea y Canadá (47-59). El tipo de toxicidad por kava parece responder a un mecanismo idiosincrásico en el que existen tres posibles mecanismos patogénicos: la inhibición del CYP450, la reducción del glutation intracelular y con menor probabilidad la inhibición de la enzima ciclooxigenasa (60). 
La Cassia angustifollia (senna) es un producto indicado para el estreñimiento debido a sus propiedades laxantes. Hasta la fecha han sido publicados 4 casos de lesión hepática secundaria a la ingesta de senna, dos de ellos con readministración positiva (61-64). La hepatotoxicidad de este producto podría ser debido a su contenido en senósidos, los alcaloides que le confieren sus propiedades laxantes y que son convertidos a antron por las bacterias intestinales, sustancia similar al dantron cuyo potencial hepatotóxico está demostrado (65).

La Valeriana officinalis (valeriana) que se encuentra en preparaciones para calmar la ansiedad, han sido señalada como posible causante de una serie de cuatro casos de hepatitis aguda, uno de los cuales cursó con insuficiencia hepática aguda, encefalopatía y fibrosis hepática (66). Así mismo se ha descrito previamente un caso de hepatotoxicidad secundaria a Aesculus hippocastanum (67). Por último, no hemos encontrado en la literatura datos previos sobre hepatotoxicidad secundaria a otras substancias como los fitoestrógenos o el chitosan.

Aunque es difícil sacar conclusiones claras o derivar en recomendaciones de una serie con tan escaso número de pacientes, sí es oportuno recordar la importancia de la identificación de todos los productos ingeridos por los pacientes con alteraciones hepáticas y recalcar la necesidad de tener en mente las sustancias naturales como posible causa de lesión hepática (68). Probablemente tendríamos un conocimiento más exacto del riesgo de estos productos si además de la detección y diagnóstico de los casos de hepatotoxicidad, se notificaran todos los casos de presunta responsabilidad de alguno de estos productos $(28,69)$.

El creciente consumo de plantas medicinales, los riesgos e incertidumbres acerca de sus efectos farmacológicos y la posibilidad de aparición de interacciones con otros medicamentos deben hacer cambiar nuestra mentalidad sobre el modo de registrar el consumo de estos productos en la historia clínica y su consideración como posibles causantes de efectos adversos (70). Es más, la posibilidad de llegar a detectar los episodios de hepatotoxicidad secundarios a sustancias herbales dependerá en gran medida de la sospecha clínica y la correcta anamnesis realizada (71). Por último, el conocimiento de que los productos de herboristería lejos de ser inocuos pueden ocasionar efectos adversos, debe animarnos a transmitir prudencia a nuestros pacientes en el consumo de estas sustancias $(72,73)$.

\section{GRUPO ESPAÑOL DE ESTUDIO DE HEPATOPATÍAS ASOCIADAS A MEDICAMENTOS (GEHAM)}

Centros clínicos participantes:

Hospital Universitario Virgen de la Victoria, Málaga (Centro Coordinador): R.J. Andrade, M.I. Lucena, Y. Borraz, M. García-Cortés, E. Ulzurrun, S. López-Ortega, A. Fernández-Castañer, M. Robles, K. Patchkoria, C. Verge,
E. García-Ruiz, E. Fernández, I. Cueto.

Hospital Torrecárdenas, Almería: M.C. Fernández, G. Peláez, R. Daza, M. Casado, J.L. Vega, F. Suárez, M. Torres, M. González-Sánchez, J. Esteban.

Hospital Universitario Virgen de Valme, Sevilla: M. Romero, A. Madrazo, R. Corpas, E. Suarez.

Hospital de Mendaro, Guipúzcuoa: A. Castiella, E.M. Zapata.

Hospital Germans Trias i Puyol, Barcelona: R. Planas, S. Anzola, J. Costa, N. López, F. García-Góngora, A. Barriocanal, A. Borras, E. Gallardo, A. Vaqué, A. Soler.

Hospital Costa del Sol, Málaga: J.M. Navarro, J.F. Rodríguez.

Hospital Central de Asturias, Oviedo: R. Pérez-Alvarez, L. Rodrigo-Saez.

Hospital Universitario San Cecilio, Granada: J. Salmerón, A. Gila.

Hospital Universitario Virgen de las Nieves, Granada: R. Martín-Vivaldi, F. Nogueras.

Hospital Carlos Haya, Málaga: M. Jiménez.

Hospital Sant Pau, Barcelona: C. Guarner, E.M. Román, G. Soriano.

Hospital Puerta del Mar, Cádiz: F. Díaz, M.J. Soria, L. Martín-Herrera, P. Rendon, M. Macias.

Hospital Virgen de la Macarena, Sevilla: A. Malcón de Dios, J.A. Durán, M. Jiménez-Sáez, A. Ruiz, J. AlanisLópez, M. Villar.

Hospital Morales Meseguer, Murcia: H. Hallal Hachem.

Hospital de Puerto Real, Cádiz: J.M. Pérez-Moreno, M. Puertas.

Hospital La Inmaculada. Huércal-Overa, Almería: H. Sánchez-Martínez.

Hospital Virgen del Rocío, Sevilla: J. Aguilar, S. Otero, F. Reina, A. Pizarro.

Hospital Juan Ramón Jiménez, Huelva: M. Ramos, T. Ferrer.

Hospital Ciudad de Jaén: E. Baeyens.

Hospital de Osuna, Sevilla: J. Pérez-Martínez.

Hospital 12 de Octubre, Madrid: T. Muñoz-Yagüe, J.A. Solís-Herruzo.

Hospital General Básico de Vélez, Málaga: F. Santalla, C. Sánchez-Robles.

Hospital Marqués de Valdecilla, Santander: F. Pons, R.

Taheri.

Hospital de Ronda, Málaga: V Díaz-Morán.

Hospital Xeral-Calde, Lugo: S. Ávila-Nasi.

Hospital de Donosti, San Sebastián: M. García-Bengoechea.

Hospital de Basurto, Bilbao: S. Blanco, P. Martínez

Hospital Gregorio Marañon, Madrid: R. Bañares.

Hospital General de Valencia: M. Diago.

Hospital Sagunto, Valencia: J. Primo, J.R. Molés.

Hospital Clínico Universitario Miguel Servet, Zaragoza: M.A. Simón.

Hospital de Laredo, Cantabria: M. Carrasco.

Hospital Clinic, Barcelona: M. Bruguera. 


\section{BIBLIOGRAFÍA}

1. Wheaton AG, Blanck HM, Gizlice Z, Reyes M. Medicinal herb use in a population-based survey of adults: prevalence and frequency of use, reasons for use, and use among their children. Ann Epidemiol 2005; $15: 678-85$.

2. Kim S, Hohrmann JL, Clark S, Munoz KN, Braun JE, Doshi A, et al A multicenter study of complementary and alternative medicine usage among ED patients. Acad Emerg Med 2005; 12: 377-80.

3. Kennedy J. Herb and supplement use in the US adult population. Clin Ther 2005; 27: 1847-58

4. Sanfélix J, Palop V, Rubio E, Martínez I. Consumo de hierbas medicinales y medicamentos. Atención Primaria 2001; 28: 311-4.

5. Ang-Lee MK, Moss J, Yuan CS. Herbal medicines and perioperative care. JAMA 2001; 286: 208-16.

6. Matthews HB, Lucier GW, Fisher KD. Medicinal herbs in the United States: research needs. Environ Health Perspect 1999; 107: 773-8.

7. Norris W, Paredes AH, Lewis JH. Drug-induced liver injury in 2007. Curr Opin Gastroenterol 2008; 24: 287-97.

8. Danan G, Benichou C. Causality assessment of adverse reactions to drugs I. A novel method based on the conclusions of international consensus meeting: application to drug-induced liver injures. J Clin Epidemiol 1993; 43: 1323-30.

9. Bénichou C, Danan G, Flahault A. Causality assessment of adverse reactions to drugs-II. An original model or validation of drug causality assessment methods: case reports with positive rechallenge. J Clin Epidemiol 1993; 46: 1331-6.

10. Benichou C. Report of an International Consensus Meeting. Criteria of drug-induced liver disorders. J Hepatol 1990; 11: 272-6.

11. Gavilan JC, Bermudez FJ, Salgado F, Peña D. Phytotherapy and hepatitis. Rev Clin Esp 1999; 199: 693-4.

12. Bruguera M, Herrera S, Lázaro E, Madurga M, Navarro M, De Abajo FJ. Hepatitis aguda asociada al consumo de Copalchi. A propósito de 5 casos. Gastroenterol Hepatol 2007; 30: 66-8.

13. Stickel F, Patsenker E, Schuppan D. Herbal hepatotoxicity. J Hepatol 2005; 43: 901-10.

14. Andrade RJ, Lucena MI, García-Cortés M. Hepatotoxicity due to herbal infusion. Gastroenterol Hepatol 2002; 25: 327-32.

15. Stedman C. Herbal hepatotoxicity. Semin Liver Dis 2002; 22: 195206.

16. Estes JD, Stolpman D, Olyaei A, Corless CL, Ham JM, Schwartz JM, et al. High prevalence of potentially hepatotoxic herbal supplement use in patients with fulminant hepatic failure. Arch Surg 2003; 138 : 852-8

17. Larrey D. Hepatotoxicity of herbal remedies. J Hepatol 1997; 26(Supl. 1): 47-51.

18. Wai CT, Tan BH, Chan CL, Sutedja DS, Lee YM, Khor C, et al Drug-induced liver injury at an Asian center: a prospective study. Liver Int 2007; 27: 465-74.

19. Jorge OA, Jorge AD. Hepatotoxicidad asociada al consume de Centella Asiatica. Rev Esp Enferm Dig 2005; 97: 115-24.

20. Edwards R. Monitoring the safety of herbal remedies. WHO project is under way. BMJ 1995; 311: 1569-70.

21. Andrade RJ, Lucena MI, Fernández MC, Peláez G, Pachkoria K, García-Ruiz E, et al. Drug-induced liver injury: an analysis of 461 incidences submitted to the Spanish registry over a 10-year period. Gastroenterology 2005; 129: 512-21.

22. Schoepfer AM, Engel A, Fattinger K, Marbet UA, Criblez D, Reichen J, et al. Herbal does not mean innocuous: ten cases of severe hepatotoxicity associated with dietary supplements from Herbalife products. J Hepatol 2007; 47: 521-6.

23. Elinav E, Pinsker G, Safadi R, Pappo O, Bromberg M, Anis E, et al Association between consumption of Herbalife nutritional supplements and acute hepatotoxicity. J Hepatol 2007; 47: 514-20.

24. Stickel F. Slimming at all costs: herbalife-induced liver injury. J Hepatol 2007; 47: 444-6.

25. Duque JM, Ferreiro J, Salgueiro E, Manso G. Hepatotoxicity associated with the consumption of herbal slimming products. Med Clin (Barc) 2007; 128: 238-9.

26. Manso G, López-Rivas L, Duque JM, Salgueiro E. Spanish reports of hepatotoxicity associated with Herbalife ${ }^{\circledR}$ products. J Hepatol 2008 ; 49: 289-90.
27. Kessler RC, Davis RB, Foster DF, Van Rompay MI, Walters EE, Wilkey SA, et al. Long-term trends in the use of complementary and alternative medical therapies in the United States. Ann Intern Med 2001; 135: 262-8.

28. Herrera S, Bruguera M. Hepatotoxicidad inducida por el uso de hierbas y medicamentos para perder peso. Gastroenterol Hepatol 2008; 31: 447-53.

29. Fernández-Castañer A, García-Cortés M, Lucena MI, Borraz Y, Peláez G, Costa J, et al. Análisis de las causas, características y consecuencias de la reexposición al fármaco o compuesto responsable de un episodio de hepatotoxicidad. Rev Esp Enferm Dig 2008; 100: 278-84.

30. Andrade RJ, Robles M, Ortiz N, Lucena MI. Reexposición en hepatotoxicidad: ¿prueba de concepto o accidente terapéutico? Rev Esp Enferm Dig 2008; 100: 255-8.

31. Federico A, Tiso A, Loguercio C. A case of hepatotoxicity caused by green tea. Free Radic Biol Med 2007; 43: 474

32. Bjornsson E, Olsson R. Serious adverse liver reactions associated with herbal weight-loss supplements. J Hepatol 2007; 47: 295-7.

33. Jimenez-Saenz M, Martinez-Sanchez M. Acute hepatitis associated with the use of green tea infusions. J Hepatol 2006; 44: 616-7.

34. Martinez-Sierra MC, Rendon P, Martín Herrera L. Hepatitis aguda tras la ingestion de té verde. Med Clin (Barc) 2006; 127: 119.

35. Bonkovsky HL. Hepatotoxicity associated with supplements containing Chinese green tea (Camellia sinensis). Ann Intern Med 2006; 144: 68-71.

36. Molinari M, Watt KD, Kruszyna T, Nelson R, Walsh M, Huang WY. Acute liver failure induced by green tea extracts: case report and review of the literature. Liver Transpl 2006; 12: 1892-5.

37. Gloro R, Hourmand-Ollivier I, Mosquet B, Mosquet L, Rousselot P, Salame E, et al. Fulminant hepatitis during self-medication with hydroalcoholic extract of green tea. Eur J Gastroenterol Hepatol 2005; 17: 1135-7.

38. Abu el Wafa Y, Benavente Fernandez A, Talavera Fabuel A, Perez Ramos MA, Ramos-Clemente JI. Acute hepatitis induced by Camellia sinensis (green tea). An Med Interna 2005; 22: 298.

39. Garcia-Moran S, Saez-Royuela F, Gento E, Lopez Morante A, Arias L. Acute hepatitis associated with Camellia thea and Orthosiphon stamineus ingestion. Gastroenterol Hepatol 2004; 27: 559-60.

40. Vial T, Bernard G, Lewden B, Dumortier J, Descotes J. Acute hepatitis due to Exolise, a Camellia sinensis-derived drug. Gastroenterol Clin Biol 2003; 27: 1166-7.

41. Pedros C, Cereza G, Garcia N, Laporte JR. Liver toxicity of Camellia sinensis dried etanolic extract. Med Clin (Barc) 2003; 121: 598-9.

42. Dueñas Sadornil C, Fabregas Puigtio S, Durandez R. Hepatotoxicity due to Camellia sinensis. Med Clin (Barc) 2004; 122: 677-8.

43. Jones FJ, Andrews AH. Acute liver injury associated with the herbal supplement hydroxycut in a soldier deployed to Iraq. Am J Gastroenterol 2007; 102: 2357-8.

44. Nota informativa de la Agencia Española del Medicamento: Suspensión de la comercialización de la especialidad farmacéutica Exolise extracto etanólico de Camelia sinensis (té verde). Ref. 2003/03.

45. Nadir A, Reddy D, Van Thiel DH. Cascara sagrada-induced intrahepatic cholestasis causing portal hypertension: case report and review of herbal hepatotoxicity. Am J Gastroenterol 2000; 95: 3634-7.

46. Pittler MH, Ernst E. Efficacy of kava extract for treating anxiety: systematic review and meta-analysis. J Clin Psychopharmacol 2000; 20: 84-9.

47. Strahl S, Ehret V, Dahm HH, Maier KP. Necrotizing hepatitis after taking herbal remedies. Dtsch Med Wochenschr 1998; 123: 1410-4.

48. Escher M, Desmeules J, Giostra E, Mentha G. Hepatitis associated with Kava, a herbal remedy for anxiety. BMJ 2001; 322: 139.

49. Kraft M, Spahn TW, Menzel J, Senninger N, Dietl KH, Herbst H, et al. Fulminant liver failure after administration of the herbal antidepressant Kava-Kava. Dtsch Med Wochenschr 2001; 126: 970-2.

50. Bujanda L, Palacios A, Silvarino R, Sanchez A, Munoz C. Kavainduced acute icteric hepatitis. Gastroenterol Hepatol 2002; 25: 434-5.

51. Campo JV, McNabb J, Perel JM, Mazariegos GV, Hasegawa SL, Reyes J. Kava-induced fulminant hepatic failure. J Am Acad Child Adolesc Psychiatry 2002; 41: 631-2

52. Wooltorton E. Herbal kava: reports of liver toxicity. CMAJ 2002; 166: 777 . 
53. Stickel F, Baumuller HM, Seitz K, Vasilakis D, Seitz G, Seitz HK, et al. Hepatitis induced by Kava (Piper methysticum rhizoma). J Hepatol 2003; 39: 62-7.

54. Russmann S, Barguil Y, Cabalion P, Kritsanida M, Duhet D, Lauterburg $\mathrm{BH}$. Hepatic injury due to traditional aqueous extracts of kava root in New Caledonia. Eur J Gastroenterol Hepatol 2003; 15: 10336.

55. Teschke R, Gaus W, Loew D. Kava extracts: safety and risks including rare hepatotoxicity. Phytomedicine 2003; 10: 440-6.

56. Humberston CL, Akhtar J, Krenzelok EP. Acute hepatitis induced by kava kava. J Toxicol Clin Toxicol 2003; 41: 109-13.

57. Gow PJ, Connelly NJ, Hill RL, Crowley P, Angus PW. Fatal fulminant hepatic failure induced by a natural therapy containing kava. Med J Aust 2003; 178: 442-3.

58. Brauer RB, Stangl M, Stewart JR, Pfab R, Becker K. Acute liver failure after administration of herbal tranquilizer kava-kava (Piper methysticum). J Clin Psychiatry 2003; 64: 216-8.

59. Thomsen M, Vitetta L, Schmidt M, Sali A. Fatal fulminant hepatic failure induced by a natural therapy containing kava. Med J Aust 2004; 180: 198-9.

60. Clouatre DL. Kava kava: examining new reports of toxicity. Toxicol Lett 2004; 150: 85-96.

61. Beuers U, Spengler U, Pape GR. Hepatitis after chronic abuse of senna. Lancet 1991; 337: 372-3.

62. Seybold U, Landauer N, Hillebrand S, Goebel FD. Senna-induced hepatitis in a poor metabolizer. Ann Intern Med 2004; 141: $650-1$
63. Sonmez A, Yilmaz MI, Mas R, Ozcan A, Celasun B, Dogru T, et al. Subacute cholestatic hepatitis likely related to the use of senna for chronic constipation. Acta Gastroenterol Belg 2005; 68: 385-7.

64. Vanderperren B, Rizzo M, Angenot L, Haufroid V, Jadoul M, Hantson $\mathrm{P}$. Acute liver failure with renal impairment related to the abuse of senna anthraquinone glycosides. Ann Pharmacother 2005; 39: 1353-7.

65. Hattori M, Namba T, Akao T, Kobashi K. Metabolism of sennosides by human intestinal bacteria. Pharmacology 1988; 36: 172-9.

66. MacGregor FB, Abernethy VE, Dahabra S, Cobden I, Hayes PC. Hepatotoxicity of herbal remedies. BMJ 1989; 299: 1156-7.

67. Takegoshi K, Tohyama T, Okuda K, Suzuki K, Ohta G. A case of Venoplant-induced hepatic injury. Gastroenterol Jpn 1986; 21: 62-5.

68. Chitturi S, Farrell GC. Hepatotoxic slimming aids and other herbal hepatotoxins. J Gastroenterol Hepatol 2008; 23: 366-73.

69. Lucena MI, Andrade RJ. Incrementando la detección de reacciones hepatotóxicas en la práctica clínica: un compromiso de calidad. Rev Esp Enferm Dig 2005; 97: 145-53.

70. Seeff LB. Herbal hepatotoxicity. Clin Liver Dis 2007; 11: 577-96.

71. Aithal GP. When is a herb a drug? Eur J Gastroenterol Hepatol 2005; 17: 391-3.

72. Lopez-Cepero JM, Lerma S, Fernandez MD, Amaya A. Hepatotoxicidad grave asociada al consumo de Noni (Morinda Citrifolia). Rev Esp Enferm Dig 2007; 99: 179-81.

73. López-Cepero JM, Fernández MD, Ferré A. Réplica. El zumo de Tahitian Noni no es hepatotóxico: nuestros comentarios. Rev Esp Enferm Dig 2007; 99: 730-41. 